\title{
Indolent, T-cell, large granular lymphocytic leukaemia in a dog presenting with severe neutropenia and an absence of lymphocytosis
}

\author{
James Elliott ${ }^{1, *}$ and Elizabeth Villiers ${ }^{2}$ \\ ${ }^{1}$ Willows Veterinary Centre \& Referral Service, Highlands Road, Solihull, B90 4NH, UK \\ ${ }^{2}$ Dick White Referrals, Station Farm, London Road, Six Mile Bottom, Cambridgeshire, CB8 OUH, UK
}

\begin{abstract}
In humans, large granular lymphocytic leukaemia (LGLL) is a low-grade, indolent lymphoproliferative disorder of large granular lymphocytes (LGL) associated with autoimmune disorders; including rheumatoid arthritis and single or multiple cytopenias; particularly neutropenia. Therapy largely centres around immunosuppression which aims to resolve the immune-mediated secondary pathology, often without eradicating the neoplastic clone. The most effective agents appear to be cyclophosphamide, cyclosporine and methotrexate. This case report describes the presentation, diagnostics, therapeutic approach and outcome of a 6 year-old Golden Retriever presenting with severe neutropenia. Chlorambucil, prednisolone and cyclosporine failed to improve the neutropenia but subsequent cyclophosphamide resulted in a sustained albeit temporary improvement in neutrophil count and the ability to withdraw prophylactic antibacterials. This case closely mirrors the diagnostics and therapeutic response in human LGLL.
\end{abstract}

Keywords: Canine, Granular lymphocytic leukaemia, T-cell.

\section{Introduction}

In humans, large granular lymphocytic leukaemia (LGLL) is a low-grade lymphoproliferative disorder characterized by the clonal proliferation of large granular lymphocytes (LGL) and recognised by the WHO (Zhang et al., 2010). This condition is often associated with autoimmune disorders; including rheumatoid arthritis and single or multiple cytopenias; particularly neutropenia (Lamy et al., 2017).

The diagnosis is often suggested by presence of an excessive proportion of circulating granular lymphocytes on haematology; though the morphology cannot be reliably distinguished from normal cytotoxic lymphocytes. Granular lymphocyte expansion can occur due to various infectious diseases including HIV infection, or post-splenectomy (Lamy et al., 2017). Flow cytometry usually demonstrates an expansion of cytotoxic $\mathrm{T}$ cells which are then confirmed as neoplastic by $\mathrm{T}$-cell receptor gene rearrangement studies showing clonality. Clonal LGL expansion arises from chronic antigenic stimulation, which promotes dysregulation of apoptosis; mainly due to constitutive activation of survival pathways including JAK/STAT, MAPK, PI3K-Akt, RasRaf-1, MEK1/ERK, sphingolipid, and NFKB (Lamy et al., 2017).

In most patients LGLL is an indolent disorder, and significant improvement of secondary cytopenias or immune disorders can be achieved with immunosuppressive agents such as corticosteroids, methotrexate, cyclophosphamide, and cyclosporin A; though trials involving more targeted therapies are underway (Lamy and Loughran, 2011; Lamy et al., 2017). There is no standard of care given the rarity of the diagnosis. Therapy often fails to eradicate the neoplastic clone (Langerak et al., 2003) and improvement in cytopenias appears to be mediated by immunosuppression; which is the mainstay of therapy. Despite this, survivals are typically prolonged with median survivals $>10$ years (Burks and Loughran, 2005; Lamy and Loughran, 2011; Lamy et al., 2017). When death does occur it appears to be secondary to overwhelming infection due to profound neutropenia; which is therefore an indication for therapeutic intervention as opposed to active monitoring only (Burks and Loughran, 2005; Lamy and Loughran, 2011). Whilst canine patients are often reported to have a LGL morphology (Tasca et al., 2009; Comazzi et al., 2011), the type of clinical presentation described above is not common in canine patients. Dogs with chronic lymphocytic leukaemia (CLL) typically exhibit a moderate-to-marked circulating lymphocytosis and absence of significant cytopenias (Adam et al., 2009; Comazzi et al., 2011).

This case report describes the diagnosis and treatment of LGLL in a canine patient with severe neutropenia and an absence of lymphocytosis which mirrors indolent LGLL in humans.

\section{Case Details}

A 6-year-old, entire male Golden Retriever presented to Willows Referral Service for investigation of neutropenia. Four weeks prior to presentation there was 
a 3-day history of mild lethargy, which appeared to self-resolve. At that time, routine haematology and biochemistry was performed which revealed a marked neutropenia $\left(0.69 ; 2.95-12.00 \times 10^{9} / 1\right)$ and moderate thrombocytopenia $\left(66 ; 148-484 \times 10^{9} / 1\right)$ so the referring veterinarian started amoxicillin-clavulanate (Synulox; Pfizer, UK) for treatment of a presumptive septic focus. The neutropenia was unchanged on repeat haematology two weeks later and so referral advised. By the time of the referral appointment the dog was clinically normal according to the owner. The dog had never left the United Kingdom.

On presentation physical examination was unremarkable and the patient was normothermic. Haematology confirmed persistent marked neutropenia $\left(0.48 ; 2.95-12.00 \times 10^{9} / 1\right)$ and mild thrombocytopenia $\left(100 ; 148-484 \times 10^{9} / 1\right)$ though smear evaluation revealed clumps and was consistent with a normal platelet count. Haematocrit was normal (38\%; 37-62\%) though noted to be at the low end of the reference range. Lymphocyte count was normal (2.47; 1.2-4.7 x 10\%/l) though blood film evaluation by a clinical pathologist reported that a significant proportion of the lymphocytes were LGL, having more voluminous and pale cytoplasm with intra-cytoplasmic magenta granules.

Flow cytometry on peripheral blood was performed to further evaluate this population of lymphocytes. This revealed a population of $\mathrm{CD} 45+\mathrm{CD} 3+\mathrm{CD} 8+$ cytotoxic T-lymphocytes, which corresponded to the LGL seen on the peripheral blood smear.

Computed tomography (CT) scan of the head and neck, thorax and abdomen with contrast revealed no abnormalities and no focus of inflammation or infection to explain the neutropenia. Fine needle aspiration of the liver and spleen were performed regardless of their normal appearance and revealed normal hepatocytes and normal splenic tissue respectively; though some LGL were noted in the splenic samples (Fig. 1) which were deemed to reflect either splenic infiltration or simply blood contamination. Urine culture was negative.

Bone marrow cytology revealed granulocytic hyperplasia (with maturation of the granulocytic series to completion) with a resultant elevated myeloid:erythroid ratio. There was an increased proportion of marrow lymphocytes (12\%; 1.7-4.9); most of which appeared to contain magenta granules similar to those in the peripheral blood (Fig. 2). Core bone marrow biopsy confirmed these findings. PCR for antigen receptor re-arrangements (PARR) on the marrow core biopsy was positive for a clonal T-cell receptor rearrangement. A diagnosis of LGLL was made with a presumptive secondary immune-mediated neutropenia. Anti-nuclear antibody (ANA) and Rheumatoid factor (RF) serology was negative.

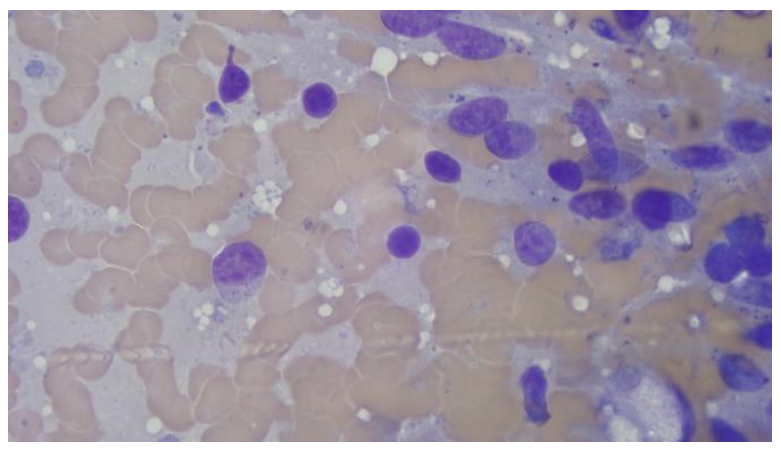

Fig. 1. Cytology of splenic fine needle aspiration at initial diagnosis showing occasional LGL.

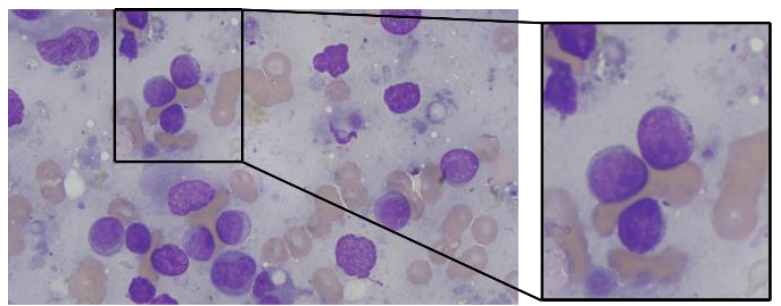

Fig. 2. Cytology of bone marrow aspiration at initial diagnosis, showing LGL in a significant proportion of lymphocytes.

PCR on EDTA blood for Leishmania, Babesia, Borrelia, Ehrlichia and Anaplasma was negative.

Two further repeat haematology samples 24 and 72 hours later confirmed neutropenia of the same magnitude and the same blood film features.

Treatment was initiated with $4 \mathrm{mg} / \mathrm{m}^{2}$ chlorambucil (Chlorambucil, Aspen, Ireland) alongside prednisolone (Prednidale 5®, Veterinary Essentials, UK) at $40 \mathrm{mg} / \mathrm{m}^{2}$, both orally and once daily. Appropriate cytotoxic handling safety was discussed with the owners. After 1, 2 and 4 weeks of therapy, neutrophil count was $0.40,0.42$ and $0.39 \times 10^{9} / 1$ respectively. Prednisolone was reduced to $30 \mathrm{mg}$ orally once daily due to marked polyuria and polydipsia (PUPD) and restlessness. Amoxicillin-clavulanate (Synulox ${ }^{\circledR}$, Zoetis, UK) was continued throughout due to neutropenia.

At week 5, the dog was deemed to be clinically normal by the owners other than some presumed steroid-related signs (panting, PUPD, polyphagia). Physical examination revealed some temporal muscle wasting and a mildly distended abdomen. Neutrophils were 0.42 $\mathrm{x} 10^{9} / 1$ with LGL present on smear evaluation. Biochemistry revealed only mild ALP elevation (168 $\mathrm{U} / \mathrm{l}$ ), presumably induced by corticosteroids. Thoracic radiographs were normal and abdominal ultrasound revealed a mildly enlarged and diffusely hyperechoic liver and a normal spleen. FNA cytology of the liver and spleen revealed only mild hepatic vacuolar change and normal splenic tissue (with some granular lymphocytes as previously) only. Ciclosporin 
(Atopica ${ }^{\circledR}$, Novartis, UK) was initiated at a dose of $5 \mathrm{mg} / \mathrm{kg}$ orally once daily instead of chlorambucil and prednisolone continued unchanged.

A lack of haematological response at week 8 (neutrophils $0.31 \times 10^{9} / 1$ with LGL on smear evaluation) prompted an increase in ciclosporin dose to $5 \mathrm{mg} / \mathrm{kg}$ orally twice daily.

At week 10 , neutrophils were $0.86 \times 10^{9} / 1$ with LGL on smear evaluation and there were no adverse effects to the higher ciclosporin dose, therefore treatment was continued. At week 12, neutrophils were $0.39 \times 10^{9} / 1$ with LGL on smear evaluation and therapy with ciclosporin was stopped.

There was still marked PUPD including nocturia. Abdominal ultrasound was performed which was unremarkable and urinalysis (cystocentesis sample, including culture) was performed and was unremarkable. Cyclophosphamide (Cyclophosphamide, Baxter Healthcare Ltd, UK) was started at a dose of 50mg orally, every other day in the morning. Prednisolone was reduced to $30 \mathrm{mg}$ orally every other day due to the magnitude of the PUPD. Furosemide was given concurrently with the cyclophosphamide to prevent drug-related sterile haemorrhagic cystitis.

At week 15, PUPD and panting had improved moderately and the dog was reported to be otherwise clinically normal at home. Neutrophils were $1.24 \mathrm{x}$ $10^{9} / 1$ with only rare LGL on smear evaluation. Therapy with cyclophosphamide was maintained and prednisolone was reduced to $20 \mathrm{mg}$ orally every other day. Amoxicillin-clavulanate was discontinued. At week 19 , neutrophil count was $6.4 \times 10^{9} / 1$ with only rare LGL on smear evaluation. No other haematological abnormalities were present. Biochemistry revealed mild ALP elevation only and free catch urinalysis was normal. Neutrophil count remained above 3.0 x $10^{9} / 1$ until week 25.

Cyclophosphamide frequency was reduced to every third day at week 25 due to a drop in neutrophil count compared the previous checks $\left(1.7 \times 10^{9} / 1\right)$ and thus a concern for possible myelosuppression. The neutrophil count had normalized 4 weeks later $\left(3.1 \times 10^{9} / 1\right)$ and every-third-day cyclophosphamide dosing was maintained.

Between week 25 and week 42, haematology was performed every 2-4 weeks and neutrophil count fluctuated between 2.6 and $5.4 \times 10^{9} / 1$ with rare LGL present on smear evaluation.

At week 44 the dog presented with a 48-hour history of anorexia and moderate abdominal pain. Biochemistry was unremarkable however haematology revealed pancytopenia (PCV 20 [37-62\%], neutrophils 0.03 [2.95-12.00 x $\left.10^{9} / 1\right]$ and platelets $\left.22\left[148-484 \times 10^{9} / 1\right]\right)$. CT of the thorax and abdomen revealed a gastric foreign body within the pylorus but was otherwise unremarkable and this was retrieved endoscopically without problem. Due to pancytopenia and concern for disease progression or a new problem, FNA of the liver, spleen and repeat bone marrow aspiration (cytology only) was performed. FNA of the liver was normal and in the spleen there was only evidence of extramedullary haematopoiesis. The bone marrow cytology revealed active and normal completion of erythropoiesis and thrombopoiesis. The myeloid lineages were also present and active but there was a dearth of mature neutrophils consistent with a maturation arrest. Occasional LGLs were seen in the marrow cytology. There was no evidence of dysmyelopoiesis or dysplastic change. Coombs' test was negative. ANA/RF serology or PARR were not repeated. Urine culture was negative. The dog recovered from anaesthesia uneventfully and immediately began eating well and was extremely bright. It was therefore assumed that the clinical signs were solely due to the foreign body and the haematological changes were incidental.

A presumptive diagnosis of immune-mediated pancytopenia was made, considered likely secondary to LGLL. Prednisolone and cyclophosphamide doses were increased to $40 \mathrm{mg}$ PO SID and 50mg PO EOD respectively and amoxicillin-clavulanate (Synulox; Pfizer, UK) was re-started at $500 \mathrm{mg}$ PO BID. The owner declined hospitalization and aggressive supportive care and the patient was discharged the morning after investigation/endoscopy as the patient was clinically normal.

After a week there was an improvement in all cell lines (PCV 29 [37-62\%], neutrophils 0.97 [2.95-12.00 x $\left.10^{9} / 1\right]$ and platelets 58 [148-484 x 109/1]) and the dog remained clinically normal other than PUPD and panting, presumably due to corticosteroids. Treatment continued but after 10 days worsening pancytopenia was evident (PCV 22 [37-62\%], neutrophils 0.04 [2.95$\left.12.00 \times 10^{9} / 1\right]$ and platelets $\left.16\left[148-484 \times 10^{9} / 1\right]\right)$ with no LGL observed on smear evaluation. Mycophenolate (250mg PO BID) was initiated as an alternative to cyclophosphamide but after 7 days of therapy the dog was lethargic, passed melaenic faces and had copious epistaxis. The owners elected for euthanasia at week 46 after initial diagnosis.

\section{Discussion}

The present case shows clinical and clinicopathological correlation with the T-cell LGLL phenotype which is seen in humans, where LGLL is divided in two main categories: T-cell LGLL and natural-killer (NK)-cell LGL (NK-LGL) leukaemia. Tcell LGLL is characterized by a clonal expansion of cytotoxic-T cells, and is an indolent lymphoproliferative disease of the elderly where approximately one third of the patients are asymptomatic at diagnosis (Lamy and Loughran, 2011; 
Lamy et al., 2017). In symptomatic patients, pathology is due to often profound secondary neutropenia where patients exhibit oral aphthous ulceration and pyrexia due to bacterial infection (Lamy et al., 2017). Severe anaemia can also be seen (Kwong and Wong, 1998). Occasional patients exhibit no clinical signs at all despite protracted and profound neutropenia. This neutropenia is likely immune-mediated and various other auto-immune conditions appear to occur concurrently including rheumatoid arthritis, systemic lupus erythematosus, Sjögren syndrome and vasculitis. Many patients have positive anti-nuclear antibody and rheumatoid factor (Lamy et al., 2017). The pathogenesis of LGLL-assciated neutropenia is not fully understood and is likely to be multifactorial, comprising both humoral and cytotoxic mechanisms (Pontikoglou et al., 2011). Since normal neutrophil survival is, at least in part, regulated by the Fas-Fas ligand apoptotic system, it has been suggested that LGLL neutropenia might be partly mediated by dysregulated Fas ligand expression. High levels of circulating Fas ligand have been consistently detected in T-LGLL serum from affected patients, possibly triggering apoptosis of neutrophils via production of secreted Fas ligand (Liu et al., 2000, 2002).

Canine CLL is a relatively rare disease, which usually affects middle-aged to older dogs (Tasca et al., 2009). It is typically characterised by an absence of, or vague clinical signs, variable magnitude of lymphocytosis and an absence of, or mild-to-moderate cytopenia(s) (Adam et al., 2009; Tasca et al., 2009). As was present in this case, a large number of canine CLL cases are characterized by larger, "non-blastic" lymphocytes with abundant cytoplasm that frequently contain azurophilic granules and thus are diagnosed as the LGL subtype of CLL (LGLL) (Tasca et al., 2009; Comazzi et al., 2011). The current case is however highly unusual in that there was a single, severe cytopenia (neutropenia) and an absence of a lymphocytosis which may mask the initial diagnosis of CLL, though the neoplastic cells were clearly circulating given their presence on blood film review. Previous studies show that up to $100 \%$ of dogs with CLL have a lymphocytosis (Tasca et al., 2009). The present case highlights the benefit of blood film review by a suitably trained professional rather than relying on analyser automated cell counts, which would not identify potentially important morphological changes. Cytopenias are typically mild in CLL cases and are less prevalent than in acute leukaemias (Adam et al., 2009), although some cases can exhibit more marked reduction in normal cell counts (Tasca et al., 2009; Comazzi et al., 2011). Unlike in the present case, previous studies have found that no dogs with CLL exhibited isolated neutropenia (Adam et al., 2009) and that neutropenia was either not marked (Comazzi et al.,
2011) or completely absent from CLL cases (Tasca $e t$ al., 2009). It has also been noted previously that anaemia is particularly common in $75-86 \%$ of CLL cases (Leifer and Matus, 1986; Tasca et al., 2009), which was not a finding in the current case. The present case is therefore very different in terms of presentation compared to classical canine CLL cases.

Cytology of a peripheral blood smear is the first step in diagnosis of LGLL in human patients and when the lymphocyte count is normal, as in this case, smears must be evaluated thoroughly for the presence of LGL. Their presence however does not prove LGLL as LGL expansion can be seen with a variety of infection and inflammatory disease (Lamy et al., 2017). Indeed LGL expansion can be seen in dogs with a variety of illnesses (McDonough and Moore, 2000) including infectious disease such as ehrlichia (Heeb et al., 2003), which highlights that further investigations are required to definitively diagnose LGLL as performed in this case. PCRs for vector-borne disease were performed in case the LGL population was reactive and this was negative. Bone marrow cytology is often not recommended in human patients as part of routine investigation as blood studies (infectious titres, cytology, flow cytometry, clonality) are more commonly performed for diagnostics. However bone marrow assessment is recommended or useful when the diagnosis is not straightforward; such as when LGL count is not significantly increased as in this case. Marrow cytology in most human patients demonstrates a significant infiltrate of LGL as in this case (>10\%) (Lamy et al., 2017). Additionally in this case, marrow evaluation was deemed necessary for diagnosis of the aetiology of the neutropenia.

Human LGLL shows a constitutive, mature postthymic phenotype. In the vast majority of cases, TLGLL shows a CD3+, TCR $\alpha \beta+, \mathrm{CD} 4-, \mathrm{CD}^{\mathrm{dim}}$, CD8+, CD16+, CD27-, CD28-, CD45R0-, CD45RA+, and CD57+ phenotype, which represents a constitutively-activated T-cell phenotype (Baesso et al., 2007). Flow cytometry is useful to discriminate Tcell LGLL from NK-LGLL and can also provide prognostic information (Lamy et al., 2017). Flow cytometry on peripheral blood in the present case confirmed the presence of the visualised LGL cells as CD45+/CD3+/CD8+ cytotoxic T-cells. Further markers were not commercially available and so not performed, however there would have been no known diagnostic or prognostic utility given the rarity of similar canine cases. Additionally in humans, treatment options are similar for T-LGLL and chronic NK-cell LGLL (Lamy et al., 2017).

An abnormal lymphocyte expansion proven with flow cytometry may simply be reactive without demonstrating clonality. In humans, documenting clonality with a PCR for T-cell receptor (TCR) gene re- 
arrangments is mandatory for the robust diagnosis of $\mathrm{T}$ cell LGLL (Lamy et al., 2017). This was positive in the current case confirming T-cell LGLL. It also proved the disease was not a chronic NK-LGLL as NK cells do not express TCR.

Immunosuppressive therapy is the foundation of treatment of human LGLL based on the rationale that leukemic LGL represent constitutively activated cytotoxic lymphocytes. First line therapy in human patients with indolent LGLL (either NK- or T-cell types) typically involves cyclophosphamide, methotrexate or cyclosporine A. Trials are lacking however and there appears to be no current standard of care (Loughran et al., 2015). There is an ongoing, randomised clinical trial to try and establish whether cyclophosphamide or methotrexate are superior as firstline therapy (Lamy et al., 2017).

Chlorambucil was initially chosen in addition to prednisolone due to its well-known utility in the treatment of CLL in dogs (Workman and Vernau, 2003). Prednisolone was used for similar reasons but also because it is typically the cornerstone of management of immune-mediated diseases in veterinary medicine (Whitley and Day, 2011). When no efficacy was realized, cyclosporine A was utilized given it is licensed for use in dogs in the UK and is a recognized treatment for LGLL in humans. However no response was seen with two different dosing regimes. Although cyclosporine is an accepted therapy for human LGLL therapy, it does seem counterintuitive to use this drug in a patient with neoplasia given the association between use of the drug and development of cancers (Blackwood et al., 2004; Schmiedt et al., 2009). The owner was fully informed about this risk prior to commencement of therapy.

The author subsequently chose cyclophosphamide in preference to methotrexate as cyclophosphamide is more commonly used in veterinary practice with a wellknown dosing and toxicity profile (Matsuyama et al., 2017) and additionally it is well known in both human and veterinary fields that methotrexate can carry a high incidence of gastrointestinal adverse effects (Curtis $e t$ al., 2016). Mycophenolate has also been shown to be effective in canine immune-mediate disease and to be well-tolerated and cost effective (Whitley and Day, 2011; Wang et al., 2013; Ackermann et al., 2017; Cummings and Rizzo, 2017).

Sterile haemorrhagic cystitis is well-recognised potential complication of cyclophosphamide therapy (Crow et al., 1977). Furosemide may reduce the incidence of cystitis when given concurrently (Charney et al., 2003; Best and Fry, 2013) and thus was suggested in this case. A small study also suggested similar benefit in low-dose or metronomic cyclophosphamide scheduling which is more akin to the treatment given in the current case (Chan et al., 2016).
Whilst there are no robust guidelines, in human chronic LGLL it is recommended that medication be tried for at least 4 months before assessing response (Lamy et al., 2017). Therefore it is possible that the chlorambucil and cyclosporine would have had more efficacy in this case if administration would have been longer. In a rare disease subtype with an unknown prognosis however it can be frustrating for owners to continue with seemingly unrewarding medications for significant time periods. Cyclophosphamide is currently one of the first-line treatments for use in human LGLL, is well tolerated by the patient and cost-effective for long-term therapy. Additionally in human LGLL a large number of patients appear to respond to cyclophosphamide despite previous lack of response to alternative agents (Bareau et al., 2010; Lamy et al., 2017). Though some authors are concerned about the use of cyclophosphamide in those patients with neutropenia due to the risk of myelosuppression (Lamy and Loughran, 2011; Moignet et al., 2014; Lamy et al., 2017). In veterinary patients this drug is similarly costeffective and widely available and was successful temporarily in current case management.

It is presumed that the neutropenia was immunemediated given the normal progression to maturity of neutrophils in the bone marrow, lack of focus of consumption and the high prevalence of immunemediated neutropenia in humans with LGLL. However confirmation of this mechanism is not possible due to the lack of commercially available molecular or immunological diagnostics available to prove this, such as anti-neutrophil antibodies. Regardless of drug therapy, immunosuppression appears to be behind the success of most humans LGLL patients. When therapy has been successful in normalizing neutrophil counts, the neoplastic clone has often not been eradicated (Langerak et al., 2003).

In this case, repeat bone marrow cytology or other invasive diagnostics were not performed to attempt to ascertain whether the burden of disease has been reduced with successful therapy. However whilst the number of LGL on blood film evaluation had subjectively reduced, there were still LGL present which might imply persistence of the neoplastic clone. It is possible however, that they are part of the normal cytotoxic T-lymphocyte repertoire.

In the longer-term, the patient in this report was intermittently mildly neutropenic. However given the sustained overall improvement in neutrophil count with cyclophosphamide and lack of success with prior agents, cyclophosphamide was continued. The major cause of morbidity in human patients is sepsis due to severe neutropenia. Therefore whilst the patient still exhibited intermittent neutropenia, counts above $1 \mathrm{x}$ $10^{9} / 1$ were considered to be "protective" against sepsis and still considered a success in case management. 
It is possible that when the haematology parameters severely declined at week 44 , the pancytopenia did not have an immune-mediated aetiology and/or was not secondary to the original diagnosis of LGLL. Indeed the Coombs' test was negative, there was no sustained improvement to immunosuppression and there was no severe infiltrate with neoplastic cells in bone marrow, liver or spleen. In fact there was a lack of significant LGL infiltrates. However a negative Coombs' test does not preclude a diagnosis of immune-mediated disease, there was a transient improvement in all cell lines after increasing the dose of prednisolone/cyclophosphamide and as already discussed in this report, LGLL typically has a low disease burden. Indeed the lack of alternative explanations for the marrow abnormalities based on imaging and liver/spleen cytology and historical diagnosis of LGLL make progressive disease most likely in the authors' opinion. The marrow cytology was consistent with an immune-mediated aetiology. Repeat RF/ANA and PARR were not performed at the time of development of pancytopenia, as whilst a positive result may have supported an auto-immune aetiology and persistent LGLL respectively, negative results would not refute either and thus would not have altered patient management.

This case demonstrates a unique presentation of canine CLL, which revealed parallels with LGLL in human oncology. Treatment with cyclophosphamide was transiently successful in improving neutrophil count, though the later development of pancytopenia suspected to be due to disease progression was the ultimate cause of the patient's demise.

\section{Acknowledgements}

The authors would like to thank Dr Stephanie Lalor and Dr Riccardo Finotello for clinical support in case management and all staff involved in the processing of samples at Dick White Referrals Clinical Pathology Service.

\section{Conflict of interest}

The authors declare that there is no conflict of interests.

\section{References}

Ackermann, A.L., May, E.R. and Frank, L.A. 2017. Use of mycophenolate mofetil to treat immunemediated skin disease in 14 dogs - a retrospective evaluation. Vet. Dermatol. 28(2), 195-e44. doi: 10.1111/vde. 12400 .

Adam, F., Villiers, E., Watson, S., Coyne, K. and Blackwood, L. 2009. Clinical pathological and epidemiological assessment of morphologically and immunologically confirmed canine leukaemia. Vet. Comp. Oncol. 7, 181-195.

Baesso, I., Pavan, L., Boscaro, E., Miorin, M., Facco, M., Trentin, L., Agostini, C., Zambello, R. and Semenzato, G. 2007. T-cell type lymphoproliferative disease of granular lymphocytes (LDGL) is equipped with a phenotypic pattern typical of effector cytotoxic cells. Leuk. Res. 31, 371-377.

Bareau, B., Rey, J., Hamidou, M., Donadieu, J., Morcet, J., Reman, O., Schleinitz, N., Tournilhac, O., Roussel, M., Fest, T. and Lamy, T. 2010. Analysis of a French cohort of patients with large granular lymphocyte leukemia: a report on 229 cases. Haematologica 95, 1534-1541.

Best, M.P. and Fry, D.R. 2013. Incidence of sterile hemorrhagic cystitis in dogs receiving cyclophosphamide orally for three days without concurrent furosemide as part of a chemotherapeutic treatment for lymphoma: 57 cases (2007-2012). J. Am. Vet. Med. Assoc. 243, 1025-1029.

Blackwood, L., German, A.J., Stell, A.J. and O'Neill, T. 2004. Multicentric lymphoma in a dog after cyclosporine therapy. J. Small Anim. Pract. 45, 259262.

Burks, E.J. and Loughran, T.P.,Jr. 2005. Perspectives in the treatment of LGL leukemia. Leuk. Res. 29, 123-125.

Chan, C.M., Frimberger, A.E. and Moore, A.S. 2016. Incidence of sterile hemorrhagic cystitis in tumorbearing dogs concurrently treated with oral metronomic cyclophosphamide chemotherapy and furosemide: 55 cases (2009-2015). J. Am. Vet. Med. Assoc. 249, 1408-1414.

Charney, S.C., Bergman, P.J., Hohenhaus, A.E. and McKnight, J.A. 2003. Risk factors for sterile hemorrhagic cystitis in dogs with lymphoma receiving cyclophosphamide with or without concurrent administration of furosemide: 216 cases (1990-1996). J. Am. Vet. Med. Assoc. 222, 13881393.

Comazzi, S., Gelain, M.E., Martini, V., Riondato, F., Miniscalco, B., Marconato, L., Stefanello, D. and Mortarino, M. 2011. Immunophenotype predicts survival time in dogs with chronic lymphocytic leukemia. J. Vet. Intern. Med. 25, 100-106.

Crow, S.E., Theilen, G.H., Madewell, B.R., Weller, R.E. and Henness, A.M. 1977. Cyclophosphamideinduced cystitis in the dog and cat. J. Am. Vet. Med. Assoc. 171, 259-262.

Cummings, F.O. and Rizzo, S.A. 2017. Treatment of presumptive primary immune-mediated thrombocytopenia with mycophenolate mofetil versus cyclosporine in dogs. J. Small Anim. Pract. 58, 96-102.

Curtis, J.R., Xie, F., Mackey, D., Gerber, N., Bharat, A., Beukelman, T., Saag, K.G., Chen, L., Nowell, B. and Ginsberg, S. 2016. Patient's experience with subcutaneous and oral methotrexate for the treatment of rheumatoid arthritis. BMC Musculoskelet. Disord. 17, 405. 
Heeb, H.L., Wilkerson, M.J., Chun, R. and Ganta, R.R. 2003. Large granular lymphocytosis, lymphocyte subset inversion, thrombocytopenia, dysproteinemia, and positive Ehrlichia serology in a dog. J. Am. Anim. Hosp. Assoc. 39, 379-384.

Kwong, Y.L. and Wong, K.F. 1998. Association of pure red cell aplasia with $\mathrm{T}$ large granular lymphocyte leukaemia. J. Clin. Pathol. 51, 672-675.

Lamy, T. and Loughran, T.P.,Jr. 2011. How I treat LGL leukemia. Blood 117, 2764-2774.

Lamy, T., Moignet, A. and Loughran, T.P.,Jr. 2017. LGL leukemia: from pathogenesis to treatment. Blood 129, 1082-1094.

Langerak, A.W., Sandberg, Y. and van Dongen, J.J. 2003. Spectrum of T-large granular lymphocyte lymphoproliferations: ranging from expanded activated effector $\mathrm{T}$ cells to T-cell leukaemia. Br. J. Haematol. 123, 561-562.

Leifer, C.E. and Matus, R.E. 1986. Chronic lymphocytic leukemia in the dog: 22 cases (19741984). J. Am. Vet. Med. Assoc. 189, 214-217.

Liu, J.H., Wei, S., Lamy, T., Epling-Burnette, P.K., Starkebaum, G., Djeu, J.Y. and Loughran, T.P. 2000. Chronic neutropenia mediated by fas ligand. Blood 95, 3219-3222.

Liu, J.H., Wei, S., Lamy, T., Li, Y., Epling-Burnette, P.K., Djeu, J.Y. and Loughran, T.P.,Jr. 2002. Blockade of Fas-dependent apoptosis by soluble Fas in LGL leukemia. Blood 100, 1449-1453.

Loughran, T.P.,Jr., Zickl, L., Olson, T.L., Wang, V., Zhang, D., Rajala, H.L., Hasanali, Z., Bennett, J.M., Lazarus, H.M., Litzow, M.R., Evens, A.M., Mustjoki, S. and Tallman, M.S. 2015. Immunosuppressive therapy of LGL leukemia: prospective multicenter phase II study by the Eastern Cooperative Oncology Group (E5998). Leukemia 29, 886-894.

Matsuyama, A., Woods, J.P. and Mutsaers, A.J. 2017. Evaluation of toxicity of a chronic alternate day metronomic cyclophosphamide chemotherapy protocol in dogs with naturally occurring cancer. Can. Vet. J. 58, 51-55.

McDonough, S.P. and Moore, P.F. 2000. Clinical, hematologic, and immunophenotypic characterization of canine large granular lymphocytosis. Vet. Pathol. 37, 637-646.

Moignet, A., Hasanali, Z., Zambello, R., Pavan, L., Bareau, B., Tournilhac, O., Roussel, M., Fest, T., Awwad, A., Baab, K., Semenzato, G., Houot, R., Loughran, T.P.,Jr. and Lamy, T. 2014. Cyclophosphamide as a first-line therapy in LGL leukemia. Leukemia 28, 1134-1136.

Pontikoglou, C., Kalpadakis, C. and Papadaki, H.A. 2011. Pathophysiologic mechanisms and management of neutropenia associated with large granular lymphocytic leukemia. Expert. Rev. Hematol. 4, 317-328.

Schmiedt, C.W., Grimes, J.A., Holzman, G. and McAnulty, J.F. 2009. Incidence and risk factors for development of malignant neoplasia after feline renal transplantation and cyclosporine-based immunosuppression. Vet. Comp. Oncol. 7(1), 4553.

Tasca, S., Carli, E., Caldin, M., Menegazzo, L., Furlanello, T. and Gallego, L.S. 2009. Hematologic abnormalities and flow cytometric immunophenotyping results in dogs with hematopoietic neoplasia: 210 cases (2002-2006). Vet. Clin. Pathol. 38, 2-12.

Wang, A., Smith, J.R. and Creevy, K.E. 2013. Treatment of canine idiopathic immune-mediated haemolytic anaemia with mycophenolate mofetil and glucocorticoids: 30 cases (2007 to 2011). J. Small Anim. Pract. 54, 399-404.

Whitley, N.T. and Day, M.J. 2011. Immunomodulatory drugs and their application to the management of canine immune-mediated disease. J. Small Anim. Pract. 52, 70-85.

Workman, H.C. and Vernau, W. 2003. Chronic lymphocytic leukemia in dogs and cats: the veterinary perspective. Vet. Clin. North Am. Small Anim. Pract. 33, 1379-1399.

Zhang, R., Shah, M.V. and Loughran, T.P.,Jr. 2010. The root of many evils: indolent large granular lymphocyte leukaemia and associated disorders. Hematol. Oncol. 28, 105-117. 